\title{
La clase de matemática desarrollada en un ambiente tecnológico: Estudiando funciones usando los deslizadores del software GeoGebra
}

\section{The mathematics class developed in a technological environment: studying functions using the GeoGebra software sliders}

\author{
Carolina Garcia, Fabiana Saldivia, Dora Maglione \\ garcia_carolina@live.com,fsaldivia@uarg.unpa.edu.ar,dmaglione@uarg.unpa.edu.ar \\ Universidad Nacional de la Patagonia Austral-Unidad Académica Rio Gallegos Av. Gdor. \\ Gregores y Piloto Rivero - Rio Gallegos - Santa Cruz - Argentina.
}

Recibido: 27/02/2019. Aceptado: 05/07/2019

\begin{abstract}
RESUMEN
En este trabajo se analizan las clases de dos docentes de diferentes cursos de nivel secundario que implementaron dos secuencias didácticas distintas para abordar el tema funciones, empleando el software dinámico Geogebra. Ambos coincidieron en usar deslizadores, y a partir del análisis a posteriori de los registros tomados y los recursos utilizados en el trabajo documental (Gueudet y Trouche, 2008) de estos docentes, observamos que los deslizadores fueron utilizados con sentidos distintos, los cuales se detallan en este artículo.
\end{abstract}

Palabras clave: Enfoque documental de la didáctica; trabajo colaborativo; desarrollo profesional; Geogebra; funciones.

\begin{abstract}
In this paper we analyze the classes of two teachers from different secondary level courses. They implemented two different didactic sequences to approach the theme of functions, using the Geogebra dynamic software. Both coincided in using sliders, and from a posteriori analysis of the records taken and the resources used in the documentary work (Gueudet and Trouche, 2008) of these teachers, we observed that the sliders were used with different meanings, which are detailed in this article.
\end{abstract}

Key words. Documentary approach to didactics; Collaborative work; Professional development; Geogebra; Functions.

\section{INTRODUCCIÓN.}

El presente ICT se genera a partir del plan de trabajo denominado "La clase matemática desarrollada en un ambiente tecnológico" llevado adelante por la becaria alumna de investigación en el proyecto 29/A372 "La inclusión del software Geogebra en las clases de matemática" en el año 2018, donde el mismo ha tenido como objetivo central problematizar el 
uso de la nuevas tecnologías en la enseñanza-aprendizaje dentro de un espacio áulico y ver el trabajo matemático de los estudiantes cuando se apropian del software.

A partir del trabajo colaborativo realizado entre el equipo de investigación y docentes de una escuela secundaria de Rio Gallegos se implementó un trayecto formativo docente para propiciar la integración del software dinámico Geogebra en las clases de matemática que llevo a estos docentes a diseñar cada uno una secuencia para implementar en los cursos donde se desempeñaban como docentes.

Consideramos el enfoque documental de la didáctica (Gueudet y Trouche, 2008, 2009) para realizar entrevistas para conocer su práctica docente habitual, como asi también los recursos (guías de trabajo, apuntes teóricos, blog, otras herramientas) que utilizan para diseñar sus clases.

En este sentido, el escrito se encuentra direccionado al análisis de los resultados obtenidos a partir de la implementación, mediante el software GeoGebra, de secuencias didácticas propuestas por docentes cuyo tema principal fue Funciones, y en particular al uso del deslizador en distintos sentidos: como parámetros de una función, por un lado, y como valores que toma la variable independiente por otro.

\section{MARCO TEÓRICO}

Tomamos como marco de referencia al enfoque documental de la didáctica introducido por los investigadores franceses Ghislaine Gueudet y Luc Trouche en el año 2008. Esta nueva línea de investigación nace con la intención de comprender el trabajo de los docentes a partir de sus interacciones con los recursos que utilizan para elaborar su proyecto de enseñanza. También consideraremos el enfoque instrumental de la didáctica (Trouche, 2004), considerando los conceptos artefacto, entendiendo por esto a una herramienta, sin considerar al usuario ni sus usos, como puede ser, por ejemplo, un software de geometría dinámica; e instrumento, que es la conjunción del artefacto y las habilidades cognitivas necesarias para construirlo. El proceso por el cual el artefacto se transforma en instrumento se denomina génesis instrumental. En dicho proceso, se distinguen, además, dos subprocesos que se diferencian entre sí según la dirección en la cual se producen las retroacciones entre el alumno y el artefacto. Por un lado, puede suceder que el artefacto influya sobre el alumno, es decir que las estrategias de resolución de los alumnos se encuentran condicionadas por las posibilidades y restricciones del artefacto (instrumentación); o puede suceder que el alumno influya sobre el artefacto, en este caso, es el conocimiento del alumno y su forma de trabajar lo que guía el modo en que utiliza el artefacto (instrumentalización).

El enfoque documental de la didáctica, (Gueudet y Trouche, 2008, 2009) por otro lado, se trata de un enfoque que tiene en cuenta al conjunto de recursos tanto antiguos, recientes o nuevos que se involucran en el trabajo docente, destacando que por recursos, se refiere a todo aquello que sea susceptible de renovar el trabajo de los docentes, los cuales pueden ser materiales, como apuntes o archivos, o pueden ser intangibles, siendo éstos de acceso un poco más difícil, ya que hacen referencia a las interacciones verbales, o no verbales, que pueden llevarse a cabo. Los recursos, son adaptados, revisados, reorganizados, a lo largo de procesos donde se articulan tanto el diseño como la implementación de los mismos, lo cual se conoce como trabajo documental; la documentación, en este sentido, se refiere tanto al trabajo que se realiza con los recursos, como a lo que se produce. El trabajo documental del docente es un proceso que impulsa la génesis documental, donde se desarrolla en forma conjunta un nuevo recurso y un esquema de uso del mismo. En este caso, en el enfoque documental, la instrumentación de la cual se habla en el 
enfoque instrumental mencionado en primera instancia, se encuentra asociada al conjunto de recursos que contribuyen a delinear la actividad didáctica del docente, mientras que la instrumentalización está relacionada con la creatividad del profesor para adaptar los recursos.

Cabe destacar que cuando se habla de "esquemas de uso del nuevo recurso" se hace referencia tanto a las normas de acción que va a considerar el docente al momento de desarrollar la clase, como a los conocimientos que pueden llegar a intervenir en la actividad del docente, los cuales pueden ser matemáticos o hacer referencia a las dificultades anticipadas, por ejemplo.

\section{CONTEXTO EDUCATIVO DONDE SE REALIZÓ LA IMPLEMENTACIÓN DE LAS SECUENCIAS DIDÁCTICAS}

Para el presente trabajo adoptamos una metodología de investigación participativa, llevando a cabo reuniones previas con docentes de una institución educativa de la ciudad de Rio Gallegos, quienes participaron activamente en la resolución de distintas situaciones problemáticas de geometría, mediante el uso del software GeoGebra, al mismo tiempo iban aprendiendo a usar el software. Además, se les propuso elaborar en conjunto alguna secuencia didáctica de interés, que involucre el uso del software y pueda ser llevada al aula; permitiéndonos participar como observadores del trabajo de los alumnos.

Ahora bien, adentrándonos en las clases que finalmente fueron observadas, cabe destacar que las secuencias didácticas implementadas en los dos cursos visitados, cuyo tema a desarrollar en ambos casos fue funciones (polinómica para uno de ellos), fueron diseñadas y propuestas, en forma particular, por los docentes que se encuentran a cargo de los mismos.

El curso en el que el Profesor Damián Fernández implementó la secuencia didáctica con la intención de introducir la noción de función, se trata de un segundo año constituido por 18 alumnos, quienes se encontraban todos presentes el día en que se fue a observar la clase. En este caso, la secuencia elaborada se desarrolló durante dos encuentros: el primero de ellos desarrollado en lápiz y papel, en el aula en la que comúnmente tienen clases; donde se introdujeron definiciones de función (con sus distintas notaciones), variables dependientes e independientes, dominio e imagen; mientras que el segundo encuentro que requería el uso del software se llevó a cabo en el laboratorio de informática del colegio, ya que permite que cada alumno trabaje individualmente en una PC. En este caso, los alumnos utilizaron por primera vez el software GeoGebra y las actividades desarrolladas, junto con la intervención docente, les permitió, en esta oportunidad, construir los conceptos de ordenada al origen, raíces de una función, además de crecimiento, decrecimiento de una función y función constante.

Por otra parte, el grupo de alumnos a cargo del Profesor Javier Nolasco, se hallaba conformado por 23 estudiantes, de los cuales estaban presentes 16 de ellos el día en que se llevó a cabo la observación. En esta oportunidad, el docente se encontraba desarrollando desde hacía tres encuentros previos (que no fueron observados) una secuencia didáctica de Funciones Polinómicas, y durante el último encuentro del tema, se hizo uso del software con el objetivo de reforzar lo trabajado hasta el momento con respecto al mencionado tema. Observando la carpeta de un alumno, se pudo determinar que lo visto durante los encuentros previos sobre funciones polinómicas fue: definición de función polinómica, dominio e imagen, orden de multiplicidad, raíces, teorema de Gauss, graficas de la función.

Cabe destacar, que este segundo grupo si había utilizado previamente el software para dibujar funciones y hallar las raíces de las mismas, por lo que algunos comandos les resultaban 
familiares. Incluso, algunos de los alumnos contaban con el software instalado en sus computadoras personales.

\section{DESCRIPCIÓN DEL TEMA DESARROLLADO IMPLEMENTANDO EL SOFTWARE}

Las secuencias didácticas llevadas al aula, fueron elaboradas y propuestas por los docentes. El docente Javier Nolasco desarrolló una secuencia didáctica que constaba de una primera parte a realizarse con lápiz y papel, con la cual el docente pretendía introducir y desarrollar el tema de funciones polinómicas, mientras que la segunda parte de la secuencia, que fuimos a observar, abordaba el tema mediante actividades que debían ser resueltas usando el software dinámico GeoGebra. En particular, lo que nos interesa analizar, tanto en esta ocasión, como con la actividad propuesta por el docente Damián Fernández para hacer uso del software, es la aplicación que cada uno de ellos hace del comando "deslizador" ubicado en la parte superior central de la pantalla del software. En la Imagen 1 se puede observar la ubicación del mencionado comando.

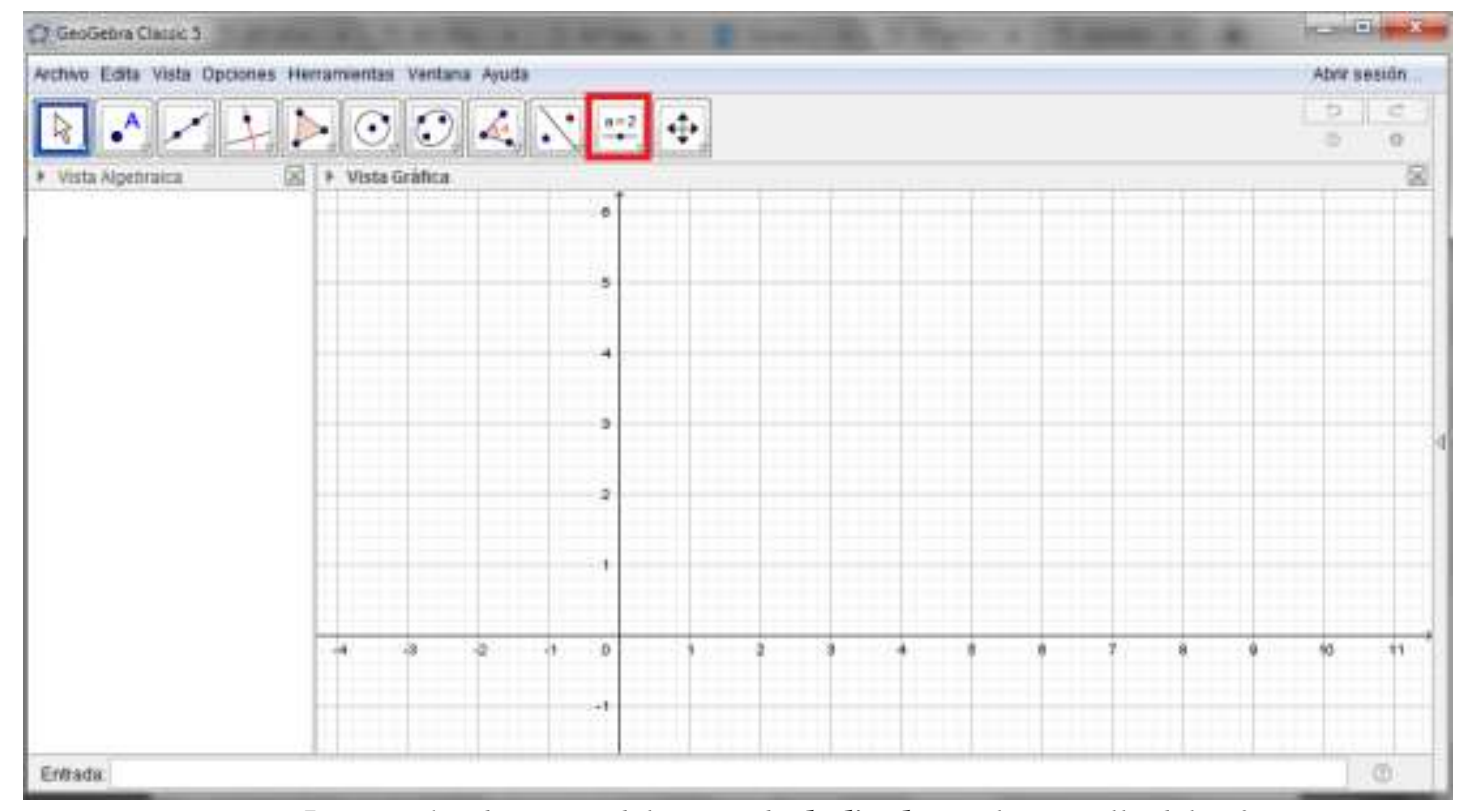

Imagen 1. Ubicación del comando deslizador en la pantalla del software dinámico GeoGebra.

\section{$\underline{\text { Las clases del profesor Javier }}$}

A continuación, presentamos una selección de actividades, llevadas adelante en un 4to año de nivel secundario, en donde se puede observar cuál es la aplicación que el docente pretende dar al deslizador: 


\section{Actividad 1: definición de los deslizadores a utilizar.}

2) Crea los deslizadores $\mathbf{a}, \mathbf{b}, \mathbf{c}$ y d con intervalo entre -20 y 20 (para ello busca intervalo escribe $-20,20$ respectivamente, debes hacerlo cuatro veces).

Esta primera actividad de la guía de repaso, tuvo como propósito que los alumnos definan los deslizadores a utilizar durante toda la guía. Para ello, se les indicó, tanto en el enunciado como en forma oral, la ubicación del comando que permite dicha creación, donde es necesario declarar datos como: nombre del deslizador, qué va a representar, intervalo en el cual el deslizador va a estar definido. En la Imagen 2 se puede observar los datos introducidos para crear los deslizadores enunciados.

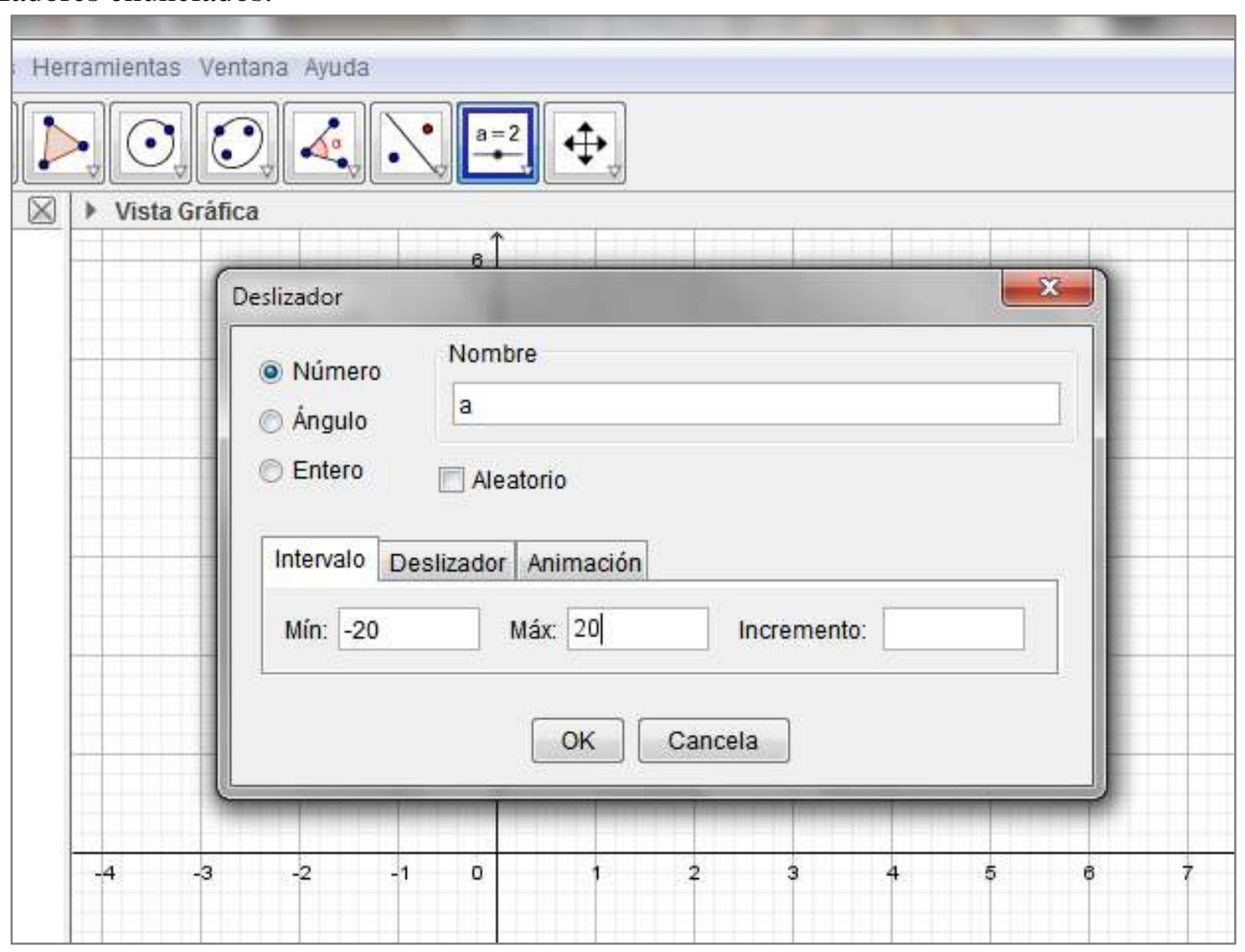

Imagen 2. Creación de los deslizadores en GeoGebra.

Actividad 2: definición de una función polinómica particular.

La segunda actividad en donde aparece el uso del deslizador, tiene como fin definir una función polinómica de grado tres. En este caso, los coeficientes de cada término de la función, tienen valor igual a los deslizadores definidos con anticipación:

3) Escribe en la barra de entrada $f(x)=a x^{3}+b x^{2}+c x+d$. Verás la expresión de la función en la vista algebraica y su representación en la vista gráfica.

Así, la función definida en la barra de entrada, que se encuentra ubicada en la parte inferior izquierda de la pantalla, queda determinada según lo pedido en la actividad, como se muestra en la Imagen 3. 


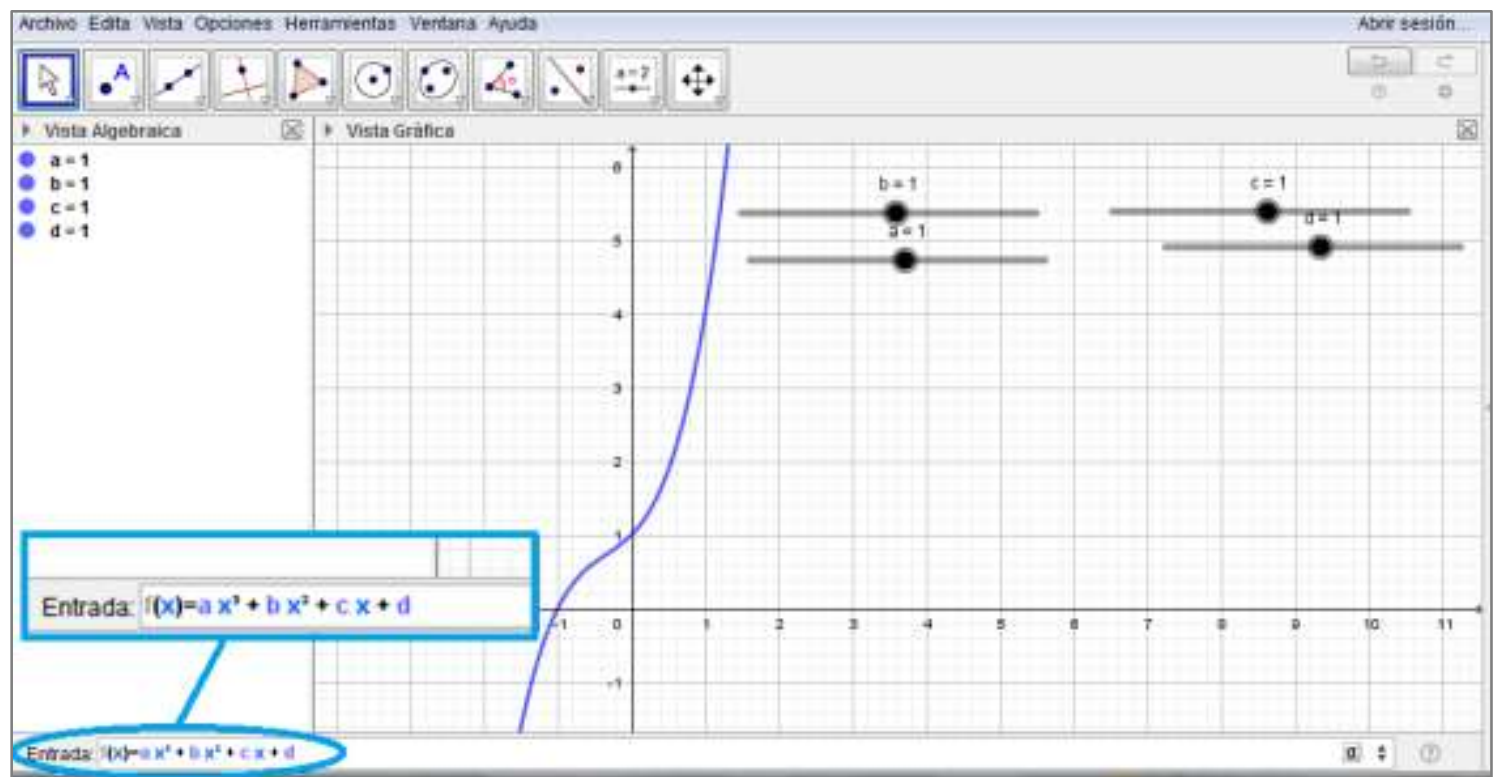

Imagen 3. Definición y representación gráfica de una función polinómica de grado tres, usando deslizadores.

Como puede observarse, a medida que se va definiendo la función en la barra de entrada, el software va graficando la función según los datos ingresados. En este primer caso, considera a todos los coeficientes con valor igual a 1 .

Actividad 3: distintos valores para cada uno de los deslizadores.

4) Cambia los valores de $\mathbf{a}, \mathbf{b}, \mathbf{c}$ y d para ello mueve el deslizador para que $\mathbf{a}=\mathbf{2}, \mathbf{b}=\mathbf{- 1}, \mathbf{c}=\mathbf{- 1 3}$ y $\mathbf{d}=-\mathbf{6}$. ¿Cuál es la expresión de f?

¿Cómo queda su gráfico?

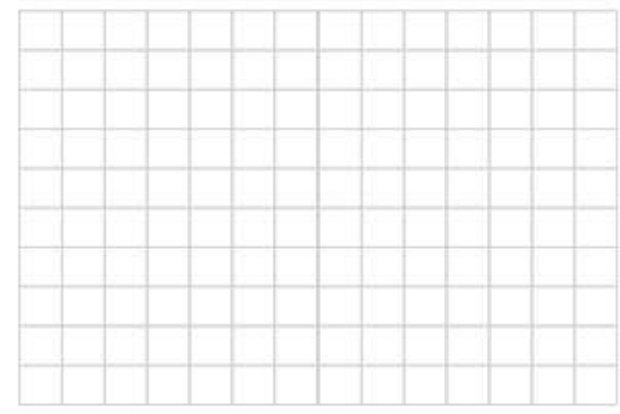

A partir de este punto, los alumnos pueden observar que a medida que mueven cada uno de los deslizadores creados, la gráfica de la función polinómica definida con anticipación comienza a modificarse.

En este sentido, las actividades de la guía enumeradas del 5 al 8, comienzan a estar orientadas a ubicar puntos en el plano considerando, ya sea el valor de la abscisa o el de la ordenada al origen, de manera tal que puedan llegar a distintas conclusiones sobre la gráfica de la función, entre las cuales podríamos destacar:

- Gráficamente, haciendo uso del software, se puede comprobar la imagen de un punto al determinar uno sobre la gráfica y con el puntero desplazarlo hasta el valor de la abscisa deseado, 
- Analíticamente, el valor de la imagen, o el valor numérico de la función polinómica, para un valor de $\mathrm{x}$ en particular, se obtiene al reemplazar dicho valor en la variable de la función definida anteriormente como $\mathrm{f}(\mathrm{x})=2 \mathrm{x}^{3}-\mathrm{x}^{2}-13 \mathrm{x}-6$.

- Si el valor de la abscisa es igual a cero, el valor número de la función se corresponde con el valor del término independiente.

- Si, por el contrario, el valor de la ordenada es igual a cero, la abscisa puede tomar tantos valores como el grado del polinomio. En este caso en particular, la abscisa puede tomar tres valores distintos cuando $\mathrm{y}=0$. A dichos valores en $\mathrm{x}$, se los llama raíces de la función.

Mientras que las actividades restantes estaban orientadas a reforzar los objetos matemáticos trabajados en los encuentros previos, referidos a hallar raíces en funciones polinómicas, ya sea gráficamente o aplicando el Teorema de Gauss, asi como también a analizar qué sucede con la gráfica de la función según cuál sea el orden de multiplicidad de las raíces. En este sentido, se realizaron representaciones graficas de tres funciones, mediante las cuales se reforzó:

- Si el orden de multiplicidad de la raíz es par, entonces la gráfica rebota en dicho valor de abscisa.

- Si el orden de multiplicidad de la raíz es impar, entonces la gráfica atraviesa el eje $\mathrm{x}$ en dicho valor de abscisa.

Cabe destacar, que el docente hizo entrega a cada uno de los alumnos, de una fotocopia en la cual se encontraban los enunciados de las 13 actividades de refuerzo a resolver durante el encuentro, la cual debía ser entregada al finalizar el mismo.

\section{Las clases del profesor Damián}

En el curso de 2do año como no conocían el software GeoGebra, la primera actividad propuesta propiciaba que los alumnos exploraran los comandos del mismo, de manera que puedan visualizar que, entre otras cosas, con él es posible dibujar puntos en el plano, graficar polígonos, e incluso se les mostró cómo construir deslizadores, definiendo uno al cual denominaron "a", que variaba entre -100 y 100 con incrementos de 1.

Dicho docente, tenía una particularidad al momento de presentar las consignas observadas. En lugar de enunciarlas, ya sea en una fotocopia para entregarles a cada uno, de escribirla en el pizarrón o de incluso proyectarla en la pantalla a la cual estaba conectado; fue haciendo un repaso oral de lo visto y trabajado el encuentro anterior. Para esto comenzó haciendo alusión a la definición de función elaborada, realizando preguntas guías de manera tal que fueran los alumnos quienes tomen la iniciativa retomando y definiendo los conceptos antes trabajados, entre los cuales también se encuentran la diferenciación entre variable dependiente e independiente, entre otros. A partir de esto, propone a los alumnos graficar haciendo uso del software, una función con la cual hayan trabajado, siendo la respuesta de un alumno, graficar: $f(x)=3 x+21$. En este momento, se les muestra cómo ingresar la función en la barra de entrada, asi como también cómo agrandar o achicar la pantalla del software para que puedan visualizar la gráfica.

Luego, las preguntas del docente se basaron en indagar si recordaban cómo ubicar un punto en el plano: “ ¿saben dibujar un punto en el plano? Por ejemplo, ¿qué significa el punto $(2,3)$ ?” y al haber respuestas positivas por parte de los alumnos, procedió a indicarles que "Los puntos de la función se pueden representar como $(x, f(x))$ ", introduciendo de esta manera el uso del deslizador, para determinar las coordenadas de un punto del plano, a partir de una función dada. 
Entre las preguntas siguientes que fue haciendo el docente de manera oral, durante el transcurso de la clase para que los alumnos vayan explorando el comportamiento de la función $f(x)=3 x+21$ mediante el software, se encuentran las siguientes:

- Si la variable dependiente vale 21, ¿Qué se aprecia en el grafico?

- ¿es la única intersección?

- Ahora, ¿qué pasa cuando intersecto al eje x? Se alcanza cuando x=-7, ¿y cuánto vale la función en ese punto?

- ¿qué pasa si tomamos valores del deslizador de menor a mayor? ¿Qué pasa con los valores de la variable dependiente?

En este punto, es posible distinguir los distintos sentidos en los que fue utilizado el comando "deslizador" por los docentes para trabajar con funciones:

- Como coeficientes de una función,

- Como coordenadas de un punto perteneciente a una función.

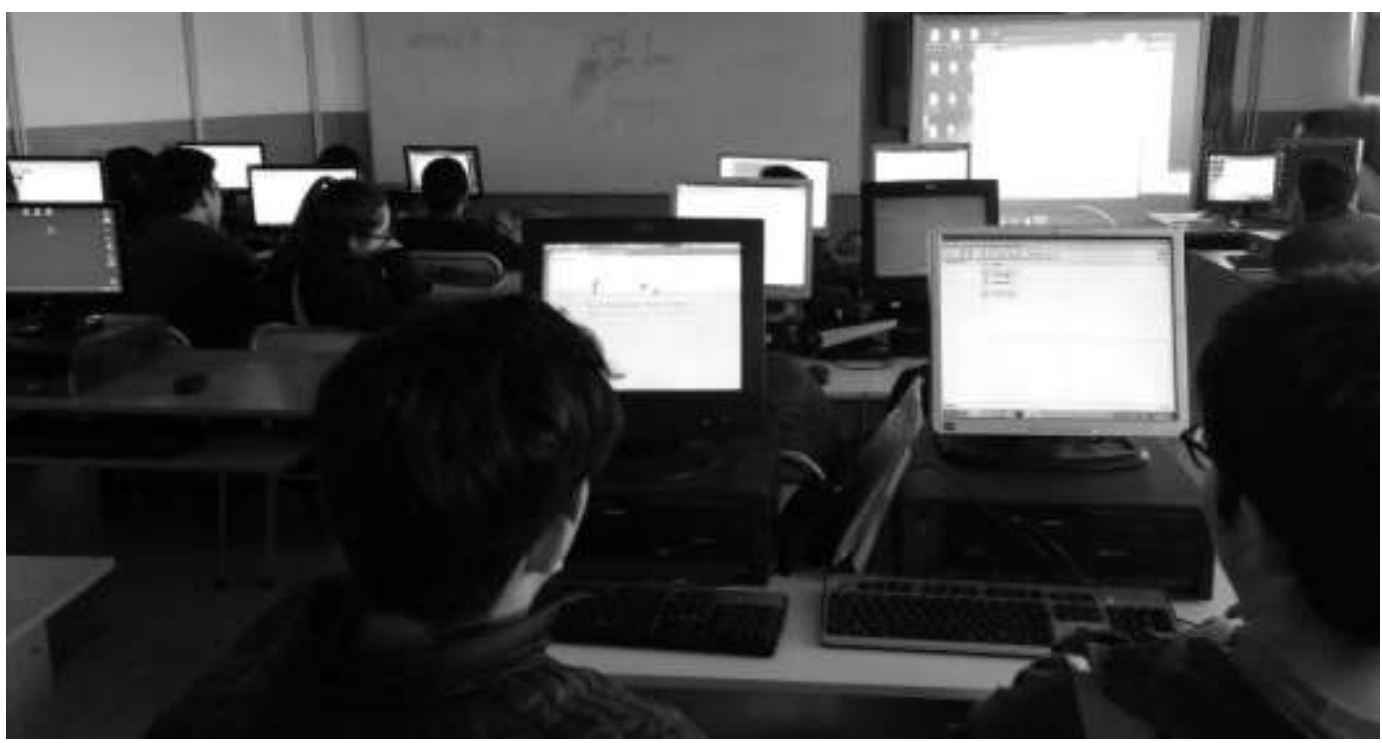

Imagen 4. Alumnos trabajando en el laboratorio de informática.

\section{LO SUCEDIDO EN LAS CLASES DONDE SE IMPLEMENTO CADA SECUENCIA}

La secuencia implementada en el segundo año de secundaria, fue observada el día 23 de octubre de 2018, mientras que la implementación en el cuarto año fue observada el día 26 del mismo mes y año.

Antes de comenzar cada clase, los docentes debían asegurarse de que las condiciones mínimas, para que los encuentros sean éxitos, estén dadas. Entendiendo por esto, a que los alumnos tengan, por lo menos, una computadora para trabajar cada dos de ellos, y que las mismas cuenten con el software instalado, además de que los docentes puedan hacer uso de un proyector conectado a una PC, de manera tal que todo el grupo pueda observar lo que él considere necesario plasmar en general para cada ocasión, como ser repasos, explicaciones de consignas o puestas en común. En general, si no se hacía previamente a que comience el encuentro, acondicionar el ambiente de trabajo llevaba un tiempo no menor a 20 minutos. Entre las dificultades que se presentaron se destaca principalmente el hecho de que las computadoras del laboratorio no tenían el programa instalado, aunque se lo hubiera instalado la clase anterior, lo cual se resolvió instalando el software nuevamente en algunos equipos. En base a esto, fue 
necesario reagrupar a los alumnos para poder llevar adelante el trabajo, formándose grupos de hasta tres integrantes en algunos casos.

Los cursos quedaron divididos en 6 y 8 grupos. En ambos casos, el trabajo grupal fue productivo. Se observaron pequeños debates entre los integrantes, quienes además en algunos casos exponían sus desarrollos e ideas en la puesta en común.

A continuación, se detallan algunas de las producciones realizadas por los grupos en los distintos encuentros.

\section{Lo sucedido en el 2do año:}

Como se indicó previamente, la primera función graficada por los alumnos haciendo uso del software, fue la función lineal definida como: $f(x)=3 x+21$, cuya grafica obtenida fue la siguiente:

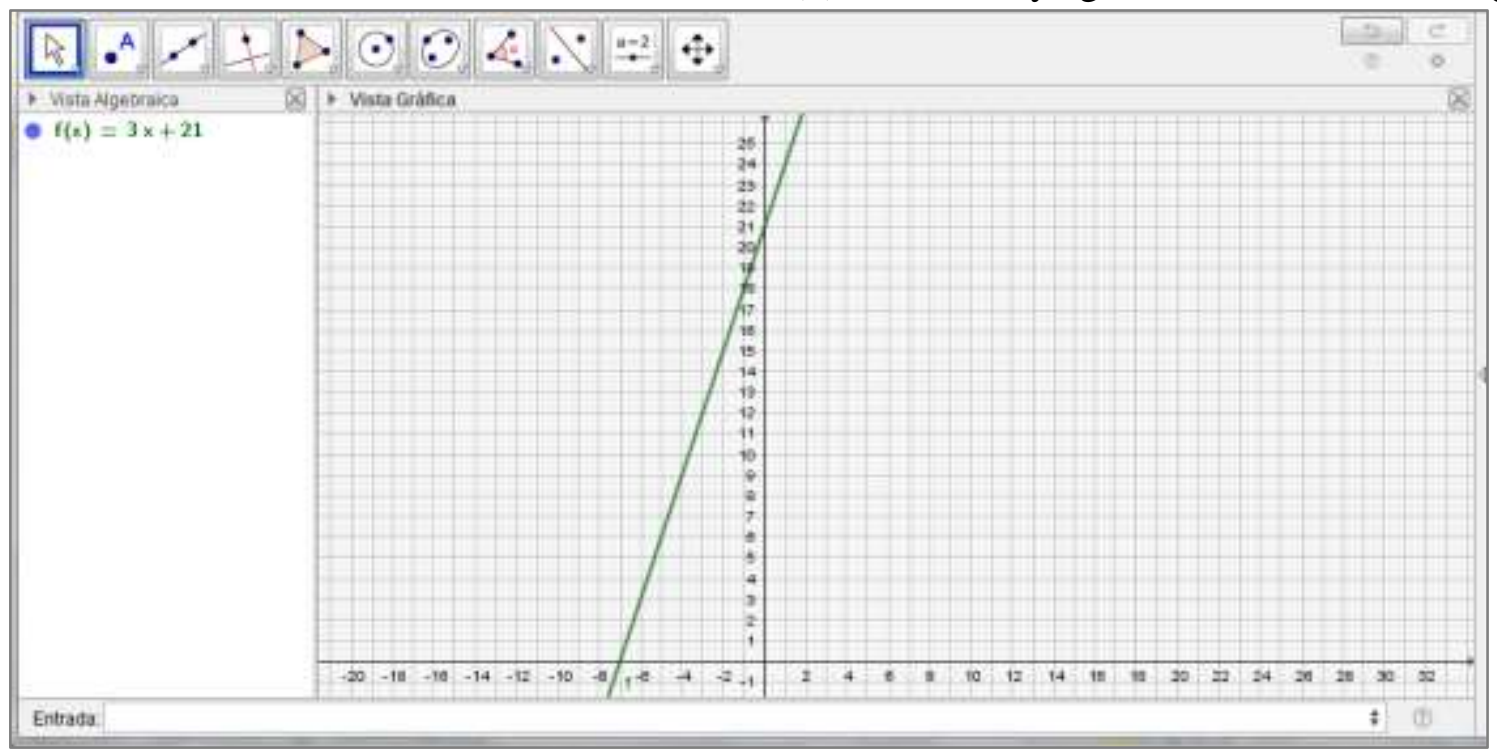

Vista grafica 1. Grafica de la función $f(x)=3 x+21$

A partir de esto, las preguntas guías realizadas por el docente para analizar la función, fueron obteniendo las siguientes producciones por parte de los alumnos:

$P:$ ¿saben dibujar un punto en el plano? Por ejemplo, ¿qué significa el punto $(2,3)$ ?

A: hay que ir del 0 a la derecha 2, del 0 a 3 para arriba y es la intersección.

$P$ : Los puntos de la función se pueden representar como $(x, \mathrm{f}(x))[\ldots]$. Por ejemplo, ¿si $x=3$

cuál sería el punto? ¿Qué tengo que hacer?

$\mathrm{f}(3)=3 \cdot 3+21$

$\mathrm{f}(3)=30$

Esto lo puedo representar a partir del punto $(3,30)$.

En este momento, se llega a la conclusión de que el punto hallado, perteneciente a la gráfica de la función lineal dada, se puede escribir como $(3, \mathrm{f}(3))$, por lo que si x toma cualquier valor, el punto a representar va a estar dado por las coordenadas (x,f(x)). De esta manera, se introdujo el uso del deslizador "a" para representar a los valores que puede llegar a tomar la variable independiente:

P: esto lo puedo hacer tomando a como entrada (a es el nombre de su deslizador). Hay que poner ....jel deslizador, como se llama?

A: a

P: y el valor de la variable dependiente. ¿Qué valor pongo?

A: $\mathrm{f}(30)$

$P: \_\mathrm{f}(30) o \mathrm{f}(\mathbf{a})$ ? 
Pudo observarse que algunos de los alumnos tuvieron problemas para ingresar esto en la barra de entrada del software, porque o han denotado A al deslizador con la letra imprenta mayúscula o a la función $\mathrm{f}$ con la letra mayúscula $\mathrm{F}$, y al querer determinar el punto indicaron coordenadas como: (A,f(a)), (a,F(a)), (3,30), ()a,f(a), por lo que no obtenían los resultados esperados. En este punto, una intervención realizada por el docente para indicar las coordenadas del punto, escribió en el pizarrón lo siguiente:

$$
\text { (nombre del deslizador, f(nombre del deslizador)) }
$$

De esta manera, cada alumno debería establecer las coordenadas, según como haya denominado al deslizador.

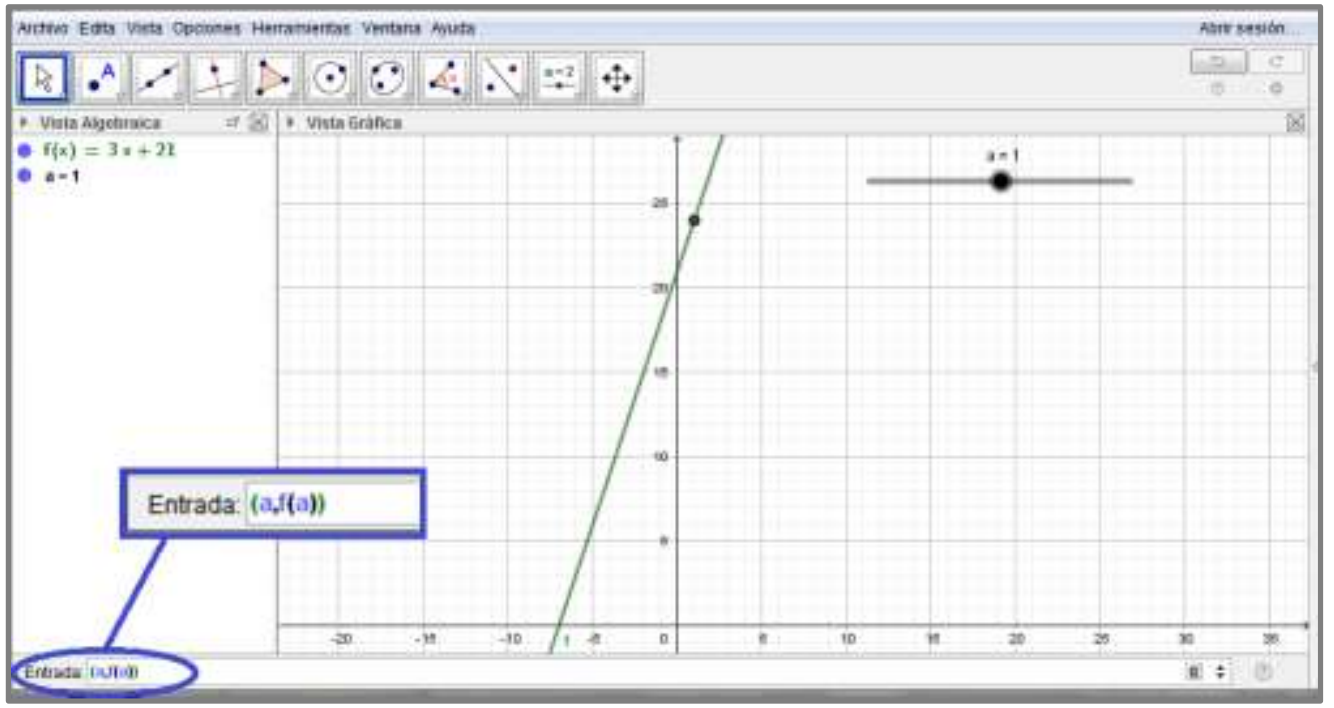

Vista grafica 2. Definición de las coordenadas de un punto, utilizando el deslizador.

Lo siguiente, fue observar cómo se va moviendo el punto sobre la recta según qué valor se le quiera asignar a la variable independiente, es decir, en este caso, según cómo se mueva el deslizador "a":

$P$ : si a vale 4, la variable dependiente vale...

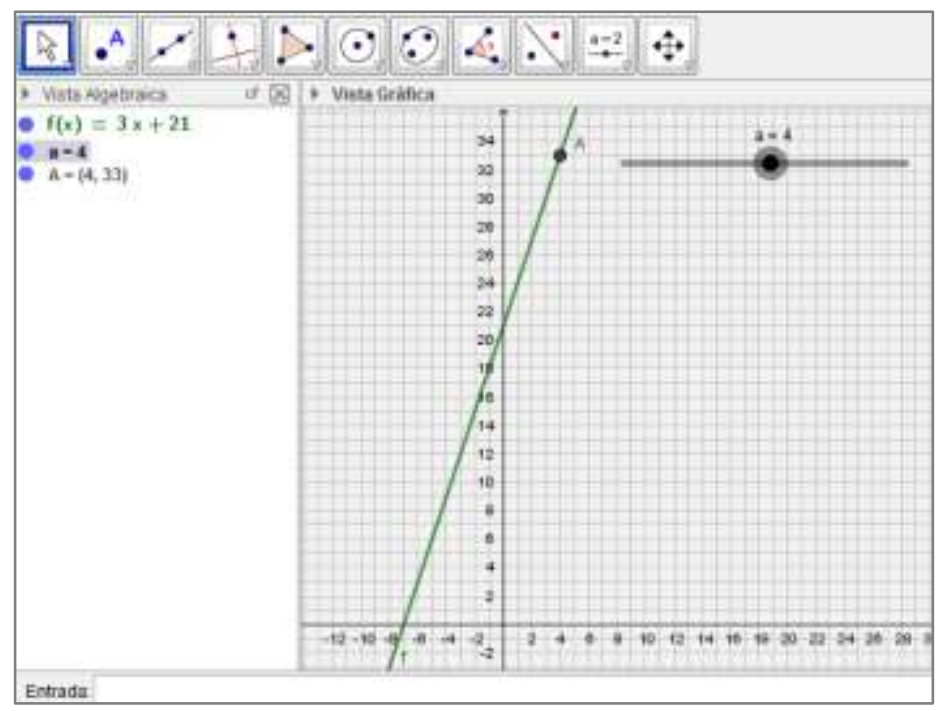

Vista grafica 3. Ubicación del punto cuya abscisa vale 4, utilizando el deslizador.

P: Si a vale 5, la variable dependiente vale... 


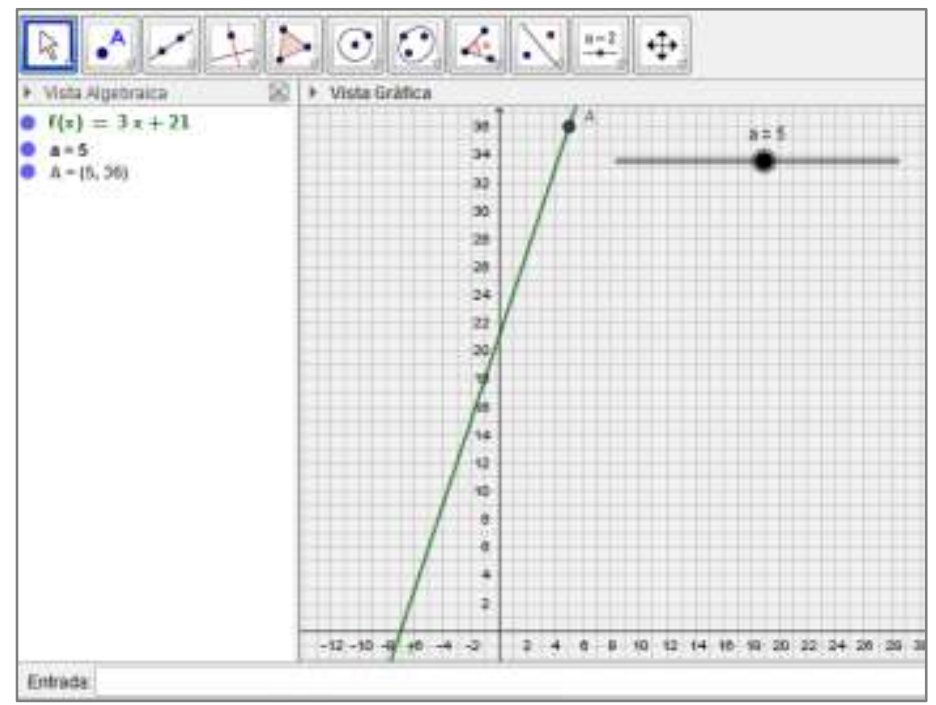

Vista grafica 4. Ubicación del punto cuya abscisa vale 5, utilizando el deslizador.

En este sentido, estableciendo distintos valores para la variable independiente (deslizador), se van obteniendo los valores que toma la variable dependiente, los cuales se pueden leer en la vista algebraica ubicada en la parte izquierda de la pantalla del software. A partir de esto, se comienza a indagar qué sucede cuando $\mathrm{x}=0$, cuando $\mathrm{y}=0$ :

$P:$ ¿Qué pasa si la variable independiente vale 0 ?

A: vale 21

P: la variable dependiente vale 21. ¿Qué se aprecia en el gráfico?

A: hay una intersección

$P:$ ¿intersección con quién?

A: eje y

Lo cual le permite al docente institucionalizar lo correspondiente a ordenada al origen, dejando por escrito en la carpeta la definición que se muestra en la Imagen 5.

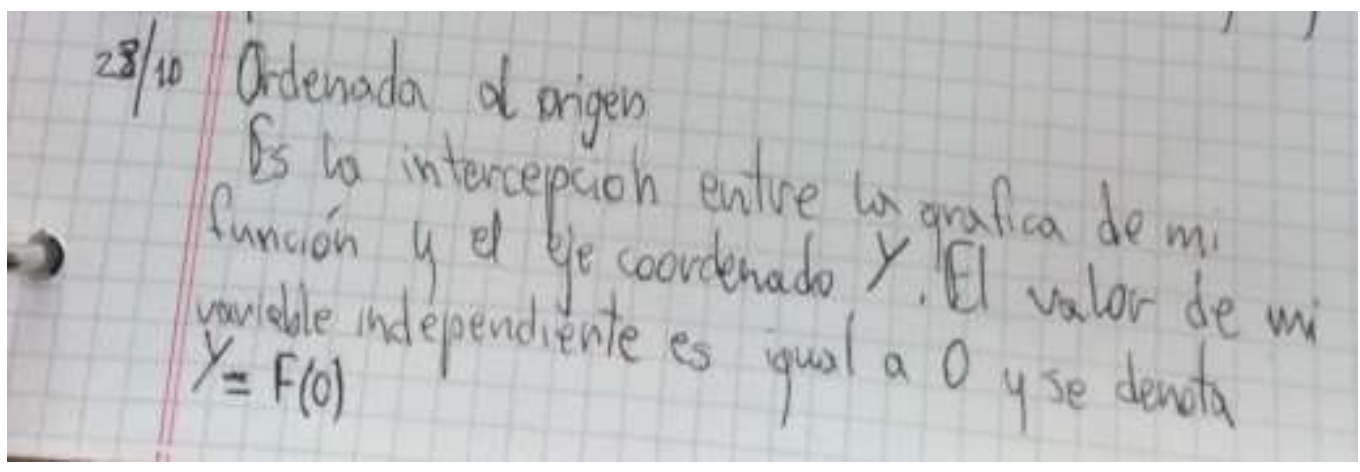

Imagen 5. Definición de ordenada al origen, extraída de la carpeta de uno de los alumnos.

De igual manera, se construye la definición correspondiente a la intersección de la función con el eje $\mathrm{x}$ :

$P$ : ¿ahora qué pasa cuando intersecto al eje $x$ ? Se alcanza cuando $x=-7, y$ ¿cuánto vale la función en ese punto?

A: 0

P: ese punto se llama raíz de la función 
En general las definiciones son construidas por los alumnos a partir de la orientación del docente, de acuerdo a lo que van respondiendo a medida que él pregunta. En este caso, lo que queda escrito en sus carpetas es lo que se muestra en la Imagen 6.

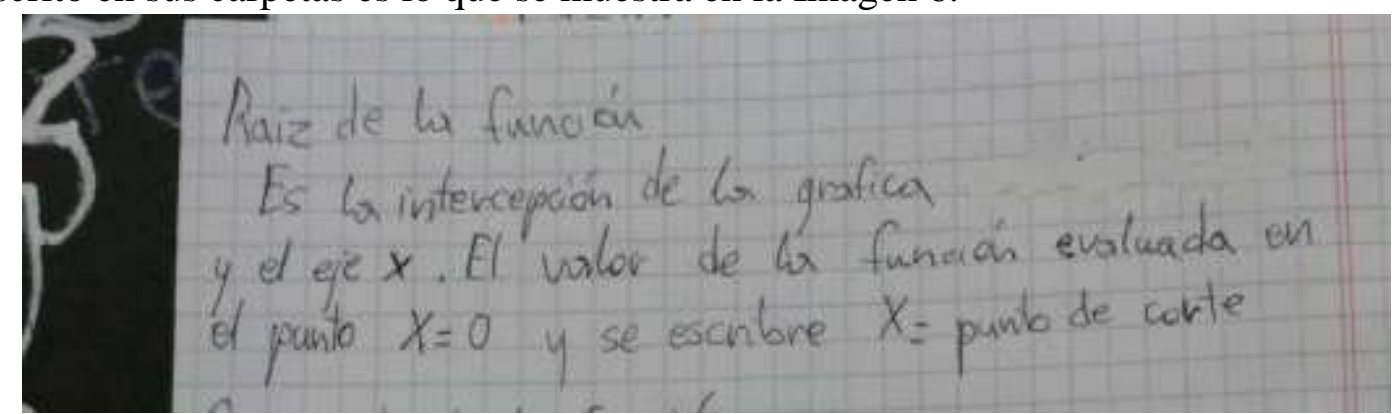

Imagen 6. Definición de raíz de una función, extraída de la carpeta de uno de los alumnos.

Por último, para hacer referencia al crecimiento o decrecimiento de la función, se plantea analizar lo que sucede cuando se mueve el deslizador desde valores pequeños a valores más grandes (se podría analizar lo mismo en sentido contrario, es decir, tomando valores de mayor a menor); en este sentido el docente pregunta:

P: ¿qué pasa si tomamos valores del deslizador de menor a mayor?, ¿Qué pasa con los valores de la variable dependiente?

Algunos alumnos dicen que los valores de la variable dependiente "suben", mientras que a otros les cuesta entender qué es lo que están diciendo. Cuando más o menos hay un consenso en el grupo, se dice que, en este caso, a medida que una variable crece, la otra también. Pero también puede suceder que mientras que una variable sube, la otra disminuya, o que no cambie; quedando escrita en las carpetas la definición que se muestra en la Imagen 7.

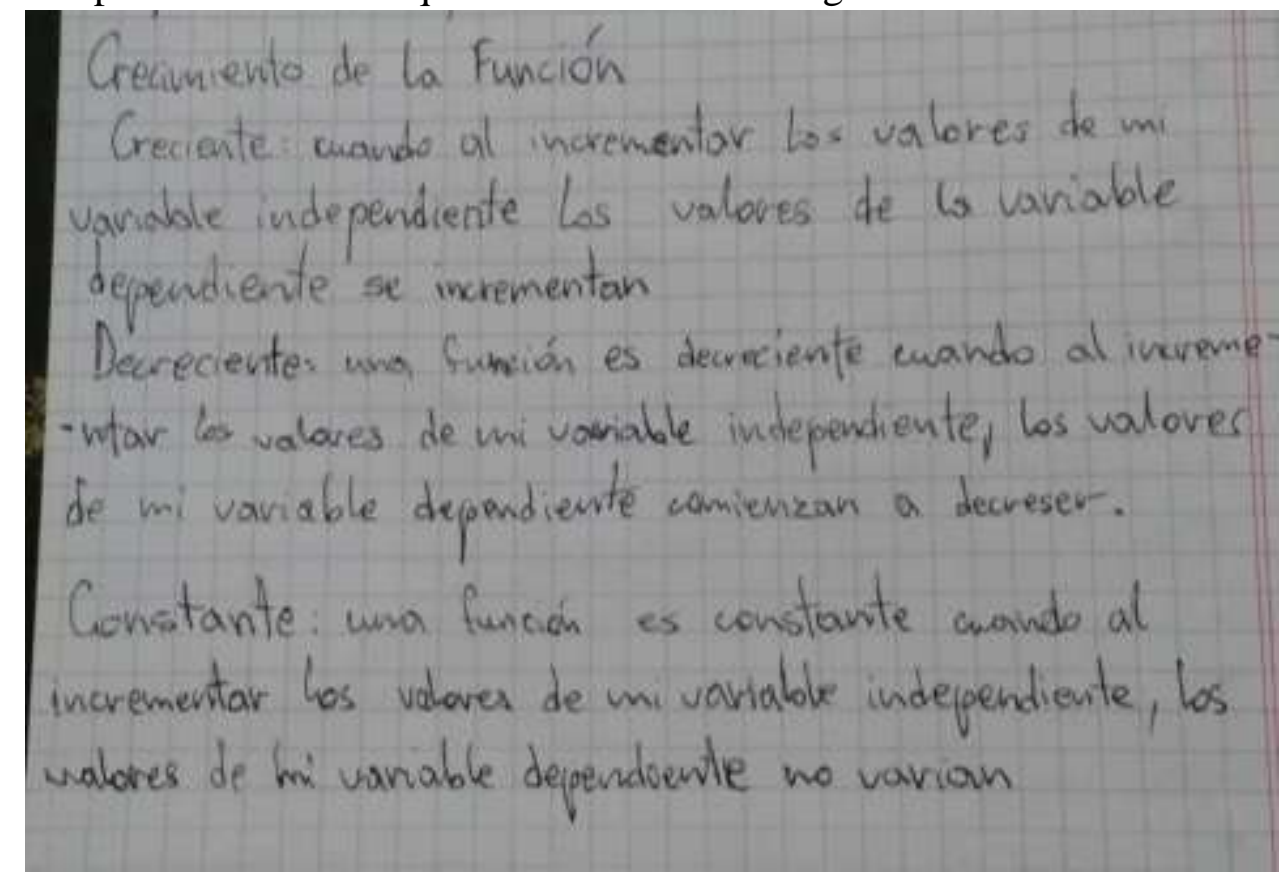

Imagen 7. Definición de crecimiento de una función, extraída de la carpeta de uno de los alumnos.

La actividad de cierre del encuentro está orientada a graficar, haciendo uso del software, una serie de funciones dadas y analizarlas indicando dominio, imagen y puntos de corte (ordenada al origen y raíz). 


\section{Lo sucedido en el 4to año.}

La resolución de la primera actividad, destinada a crear los deslizadores, fue guiada por el docente, ya que es la primera vez que los alumnos hacen uso del correspondiente comando en el software. En esta ocasión todos los alumnos crean los cuatro deslizadores (a, b, c y d) sin problemas, salvo un único grupo que les asignó otros nombres, por lo que luego, tuvieron problemas al definir y graficar la función polinómica de grado tres solicitada en la actividad número dos.

En este sentido, algunos de los errores de los alumnos, observados en la producción de la segunda actividad fueron:

- El grupo que puso otros nombres a los deslizadores, no lograba hacer la gráfica de la función polinómica definida como $\mathrm{f}(\mathrm{x})=\mathrm{ax}^{3}+\mathrm{bx}^{2}+\mathrm{cx}+\mathrm{d}$.

- Hubo quien incluyó los coeficientes a y b dentro de un paréntesis, definiendo a la función como: $f(x)=(a x)^{3}+(b x)^{2}+c x+d$.

- Hubo quien sólo definió a la función dada en la barra de entrada como: "= $\mathrm{ax}^{3}+\mathrm{bx}^{2}+\mathrm{cx}+\mathrm{d}$ ", y por algún motivo no fue interpretado por el software, de manera que no graficaba la función deseada.

- Una de las alumnas obtuvo como representación gráfica de la función definida, una recta, debido a que no determinó los exponentes de cada una de las variables. Simplemente definió a la función como: $\mathrm{f}(\mathrm{x})=\mathrm{ax}+\mathrm{bx}+\mathrm{cx}+\mathrm{d}$

Una vez que los alumnos tuvieron definida y graficada la función polinómica de la segunda actividad en el software, se procedió a modificar los valores de los coeficientes, tal y como se pide en la tercera actividad, de manera que la gráfica de la función, en esta ocasión, considerando que $\mathbf{a}=\mathbf{2}, \mathbf{b}=-\mathbf{1}, \mathbf{c}=-\mathbf{1 3}, \mathbf{d}=-\mathbf{6}$, es la que se muestra en la vista grafica 5 .

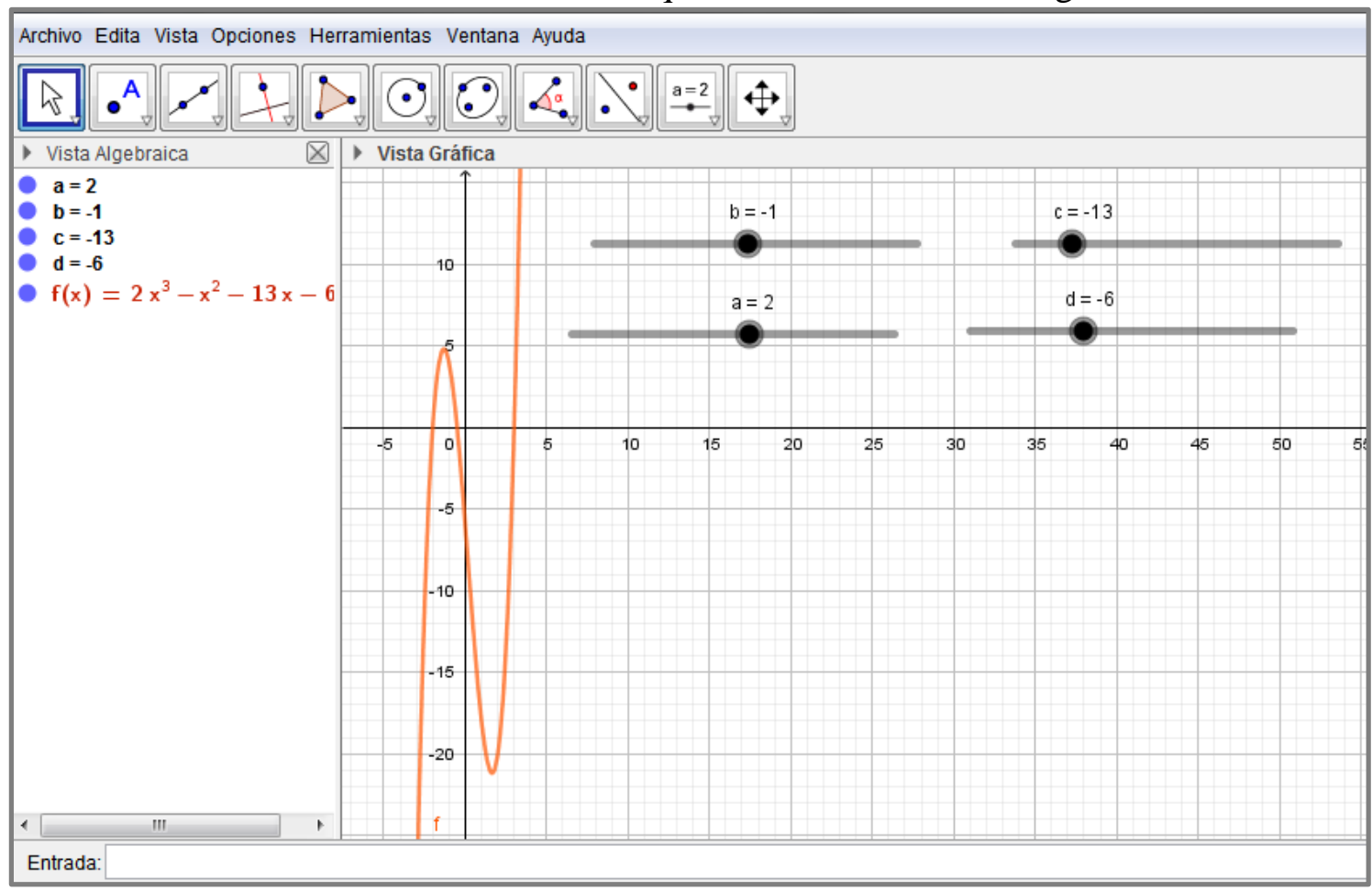

Vista grafica 5. Grafica de la función $f(x)=2 x^{3}-x^{2}-13 x-6$. 
En general, la resolución de esta actividad no tuvo muchos inconvenientes y fue resuelta por todos los presentes. En la carpeta de uno de los alumnos, la resolución de este inciso quedó registrada como se muestra en la imagen 8 .

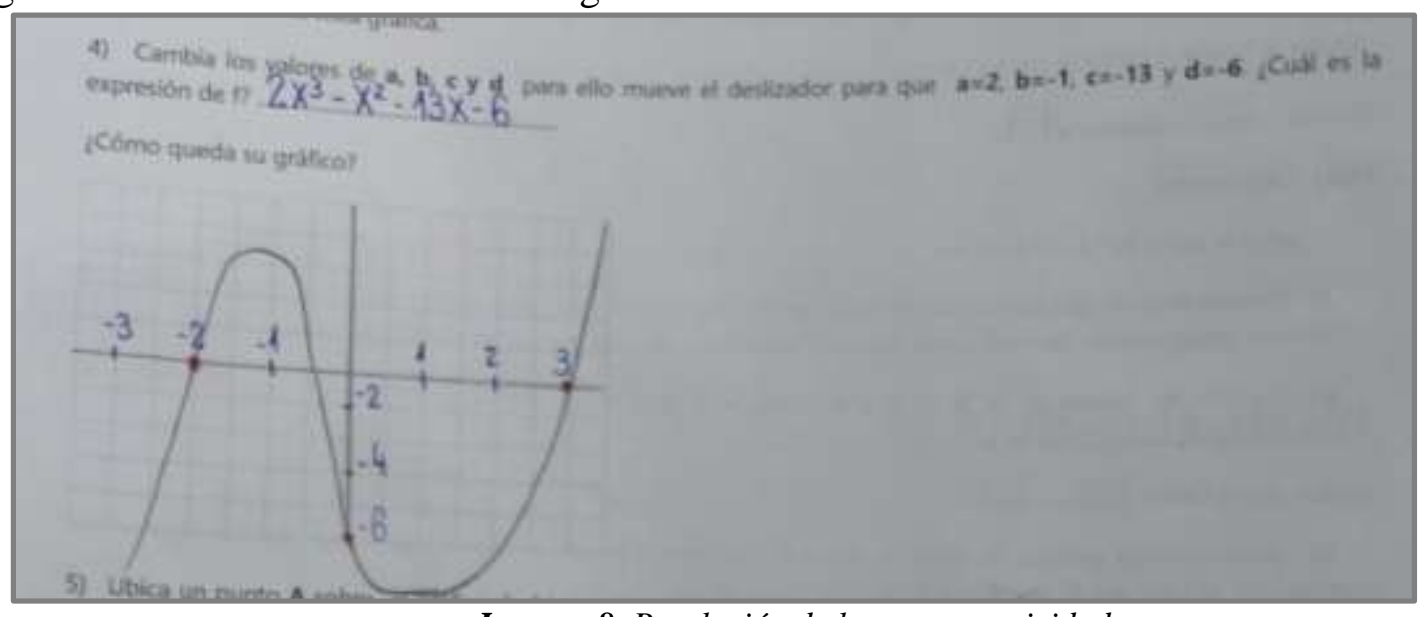

Imagen 8. Resolución de la tercera actividad.

En este caso en particular, puede observarse que el alumno determino correctamente la expresión de las funciones, pero no estableció una escala adecuada para hacer uso del recuadro designado por el docente y graficar en lápiz y papel lo que la pantalla del software mostraba. Por lo que simplemente pudo indicar en el eje coordenado los puntos de corte de la función (raíces y ordenada al origen) y en base a ello realizó una gráfica aproximada, sin considerar los máximos y mínimos de la misma. Si bien en el recuadro designado no era posible graficar utilizando la escala elegida por el alumno, podría haberse considerado resolver la actividad en hoja aparte, de manera que la representación se asemeje un poco más a la correcta.

Otro alumno, en cambio, plasmó en su hoja la resolución de esta misma actividad como se muestra en la imagen 9 .

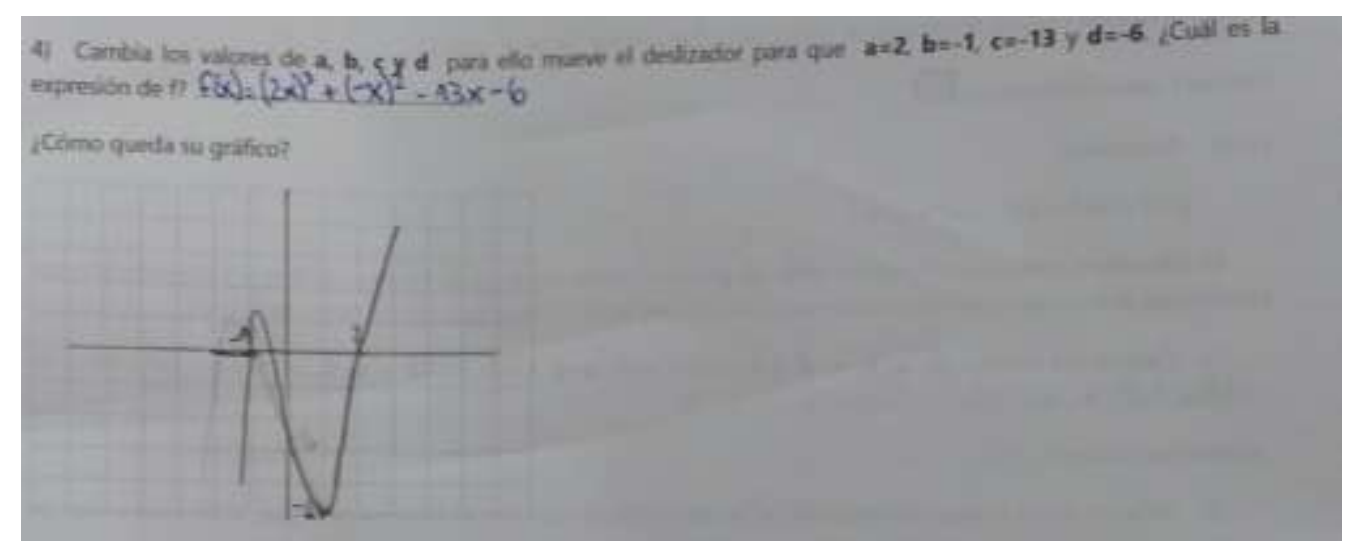

Imagen 9. Resolución de la tercera actividad, extraída de la carpeta de un segundo alumno.

Este segundo alumno, como puede observarse, fue uno de los que definió a la función de la actividad dos de manera incorrecta como $\mathrm{f}(\mathrm{x})=(\mathrm{ax})^{3}+(\mathrm{bx})^{2}+\mathrm{cx}+\mathrm{d}$, por lo que la gráfica de la función obtenida en el software para esta tercera actividad, al modificar el valor de los deslizadores, fue la que se muestra en la vista grafica 6. 


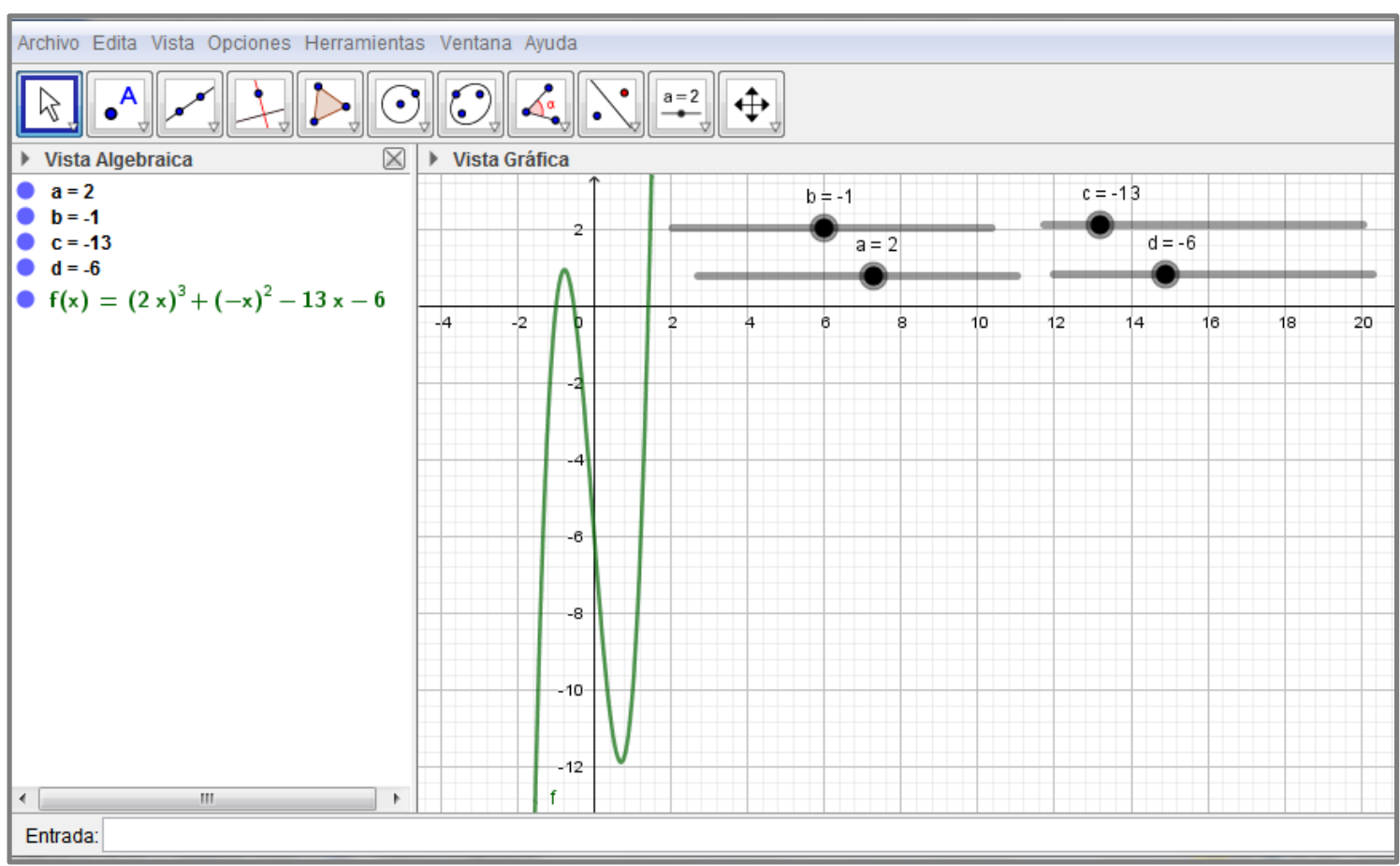

Vista grafica 6. Gráfica de la función definida por un alumno como $f(x)=(2 x)^{3}+(-x)^{2}-13 x-6$.

Dicha grafica fue plasmada por el alumno en su carpeta de manera aproximada, ya que si se verifica, al utilizar el comando "raíces" del software, se puede apreciar que la gráfica corta al eje de las abscisas en $x_{1}=-1, x_{2}=-0,53, x_{3}=1.41$, mientras que el eje de las ordenadas es cortado en $y=-6$, además de que el punto máximo no alcanza al valor 1 en el eje de las ordenadas, y el mínimo es menor a -11, también en el eje de las ordenadas. Sin embargo, no es la gráfica correcta solicitada, debido al error de definición de la función antes mencionado.

El docente tomaba conocimiento de estos errores en la producción de los alumnos al pasar por los bancos, de manera que realizaba las devoluciones pertinentes para orientarlos en su trabajo de manera individual; mientras que la puesta en común se encontraba orientada a establecer entre todos la resolución correcta de los ejercicios, o incluso a resolver en forma grupal algún inciso, más que nada aquellos destinados a hallar en forma analítica las raíces de alguna función polinómica indicada, mediante la aplicación del Teorema de Gauss. Para la resolución de ejercicios de este tipo, el docente hizo uso de una planilla Excel, diseñada por él mismo, la cual facilitaba y agilizaba los cálculos. En este sentido, un grupo particular de alumnos se mostraban muy atentos a las resoluciones en conjunto con el docente frente al pizarrón y cuando podían aportar información o encontrar algún error lo hacían inmediatamente. 


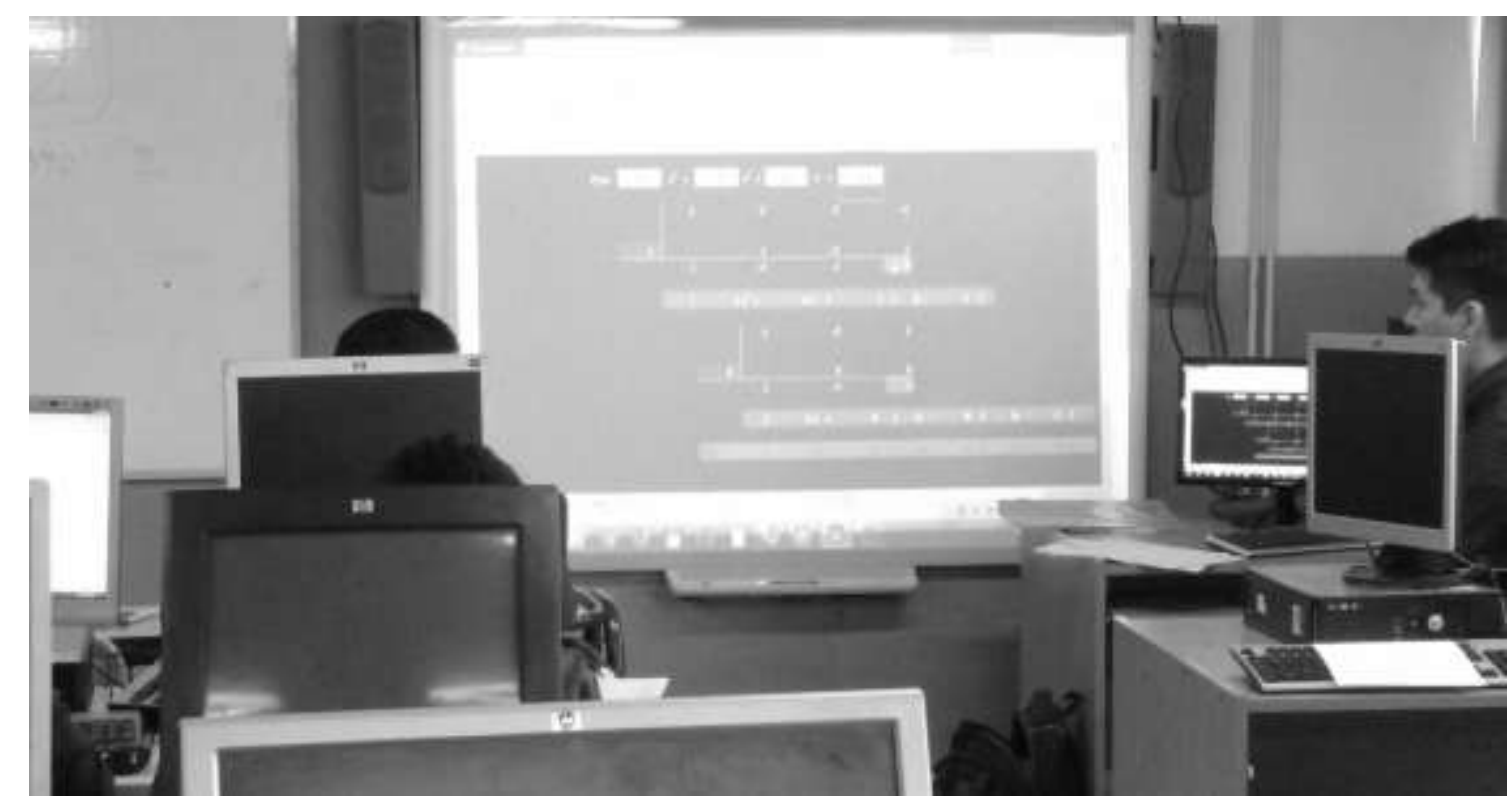

Figura 8. Momento en que se hallan raíces de una función polinómica de forma grupal, haciendo uso de la planilla diseñada por el docente.

\section{A MODO DE CIERRE}

El presente trabajo se ocupó de ahondar sobre el uso del comando "deslizador" del software dinámico GeoGebra, y a partir de ello el surgimiento y la utilización del símbolo como una herramienta matemática. En este sentido, la forma de trabajo adoptada en el presente ICT fue realizar diversos encuentros dentro de espacios áulicos, con el fin de observar la implementación de una serie de actividades diseñadas por los docentes, las cuales implicaban el uso del TIC, con el fin de propiciar una mejor aprehensión de los objetos matemáticos.

En el análisis fue importante tener presente de forma continua la noción del sentido de símbolo para intentar comprender el trabajo docente y los posibles posicionamientos en cuanto a su utilización; es asi como consideramos oportuno retomar lo establecido por Abraham Arcavi (1994) en "Symbol sense: informal sense-making in Formal Mathematics" donde establece:

"(...) el sentido de símbolo incluye:

- Una comprensión de y un sentir estético del poder de los símbolos: cómo y cuándo los símbolos pueden y deben ser utilizados a fin de mostrar relaciones, generalizaciones y pruebas que de otra manera están ocultas, escondidas o son invisibles.

- Un sentimiento de cuándo conviene abandonar a los símbolos en favor de otro tipo de enfoques o acercamientos a fin de poder lograr progresos ante un problema, o con el objetivo de encontrar una solución o representación más fácil y más elegante."

Teniendo en cuenta esto, es posible destacar la diversidad en cuanto al abordaje de una misma temática, haciendo referencia a las secuencias didácticas implementadas por los docentes, donde se pudo evidenciar que, si bien ambos decidieron utilizar deslizadores en sus actividades, el sentido dado en su implementación, fue distinto, pero no por ello menos válido. En este sentido, podemos mencionar el proceso que llevo a cabo uno de los docentes, por el cual los alumnos fueron capaces de construir junto a él, el porqué de la aparición de los símbolos para representar una situación dada, en este caso en particular, las coordenadas de un punto perteneciente a una 
función. En cambio, en la secuencia del otro docente solo se favoreció el uso del software. Si bien resulta interesante hacer uso de los comandos del software, las actividades propuestas en esta segunda ocasión podrían haber sido resueltas de igual manera sin que surja el uso de los deslizadores, simplemente definiendo en la barra de entrada las distintas funciones con las cuales trabajar.

Otra cosa que se puede concluir, de lo ocurrido en las clases de 4to año, es el uso que el docente hizo de los símbolos en lápiz y papel en una instancia previa a la experiencia en el laboratorio de informática y que no retomó durante esta última ocasión. Pudiéndose apreciar que, en la resolución en lápiz y papel, el docente propiciaba distintas formas de escritura de una misma función, pero en el trabajo realizado con el software esto no fue considerado.

Por otro lado, observamos coincidencias en el uso del deslizador para definir el dominio de las funciones. Esto nos mostró las limitaciones que conlleva el uso del deslizador, generando las siguientes preguntas: ¿se podrían hallar todas las raíces a partir de la restricción del dominio al definir el deslizador en un intervalo cerrado? ¿Qué otro uso se puede hacer al definir subfamilias de funciones polinómicas de grado 3? ¿Estamos trabajando otra vez con restricciones? ¿El docente tiene que explicitar estas restricciones?

Para finalizar, ha de resultar interesante poder plantear la necesidad o conveniencia de la utilización oportuna del símbolo para el trabajo matemático, ya que en ocasiones en lugar de favorecer o aportar algo nuevo puede entorpecer o no ser un aporte significativo. Ahora bien, no se desconoce la relevancia de seguir profundizando nuestra formación sobre este campo, asi como también la aplicación de dicha herramienta en el software dinámico GeoGebra.

\section{AGRADECIMIENTOS}

Se agradece a los docentes Damián Fernández, Javier Nolasco y Miguel Ángel Robles, quienes conformaron junto al equipo de investigación un grupo de trabajo colaborativo, como así también a los alumnos que nos permitieron llevar a cabo la investigación. 


\section{REFERENCIAS BIBLIOGRÁFICAS.}

ARCAVI A. (1994). Symbol sense: Informal sense-making in Formal Mathematics. For the Learning of Mathematics, 14, 315 - 329.

GUEUDET, G.; TROUCHE, L. (2008). Du travail documentaire des enseignants : genèses, collectifs, communautés. Le cas des mathématiques. Education et didactique, 2(3), 7 33. https://doi.org/10.4000/educationdidactique. 342

GUEUDET, G.; TROUCHE, L. (2009). Towards new documentation systems for mathematics teachers? Educational Studies in Mathematics, 71(3), 199-218. https://doi.org/10.1007/s10649-008-9159-8

TROUCHE, L. (2004). Managing the complexity of human/machine interactions in computerized learning environments: guiding students' command process through instrumental orchestrations. International Journal of Computers for Mathematical Learning, 9, 281-307. https://doi.org/10.1007/s10758-004-3468-5 